\title{
Screening and Functional Analysis of Hub MicroRNAs Related to Tumor Development in Colon Cancer
}

\author{
Dong-Hu Yu, ${ }^{1,2}$ Wei Li, ${ }^{3}$ Jing-Yu Huang, ${ }^{4,5}$ Xiao-Ping Liu, ${ }^{2}$ Chi Zhang, ${ }^{6}$ Xiao-Lan Ruan $\mathbb{D},{ }^{7}$ \\ and Sheng $\mathrm{Li}$ iD \\ ${ }^{1}$ Department of Biological Repositories, Human Genetics Resource Preservation Center of Hubei Province, \\ Zhongnan Hospital of Wuhan University, Wuhan 430071, China \\ ${ }^{2}$ The Second Clinical College, Wuhan University, Wuhan 430071, China \\ ${ }^{3}$ Department of Oncology, The First People's Hospital of Tianmen, Tianmen 431700, Hubei Province, China \\ ${ }^{4}$ Department of Thoracic Surgery, Zhongnan Hospital of Wuhan University, Wuhan 430071, China \\ ${ }^{5}$ Hubei Key Laboratory of Tumor Biological Behaviors \& Hubei Cancer Clinical Study Center, Wuhan 430071, China \\ ${ }^{6}$ Department of Gastrointestinal Surgery, Hubei Cancer Hospital, Tongji Medical College, \\ Huazhong University of Science and Technology, Wuhan 430071, China \\ ${ }^{7}$ Department of Hematology, Renmin Hospital of Wuhan University, Wuhan 430071, China
}

Correspondence should be addressed to Xiao-Lan Ruan; 344901800@qq.com and Sheng Li; lisheng-znyy@whu.edu.cn

Dong- $\mathrm{Hu} \mathrm{Yu}$ and Wei Li contributed equally to this work.

Received 17 June 2019; Accepted 8 August 2019; Published 23 January 2020

Academic Editor: Ernesto S. Nakayasu

Copyright (C) 2020 Dong-Hu Yu et al. This is an open access article distributed under the Creative Commons Attribution License, which permits unrestricted use, distribution, and reproduction in any medium, provided the original work is properly cited.

\begin{abstract}
Various microRNAs (miRNAs) are of importance in the development of colon cancer, but most of the mechanisms of the miRNAs are still unclear. In order to clarify the hub miRNAs and their roles in colon cancer development, GSE98406 was used to screen hub miRNAs by bioinformatics analysis. 46 DE-miRNAs (14 were upregulated and 32 were downregulated) and 1738 target genes of DE-miRNAs were ascertained. miRNAs-gene-networks and miRNAs-GO-networks were built to get more knowledge about the function of candidate miRNAs. After validation, three miRNAs (miR-17-5p, miR-182-5p and miR-200a-3p) were recognized to be hub miRNAs associated with the progression of colon cancer. More importantly, the hub miRNAs and the putative targets genes might be new diagnostic and therapeutic targets for colon cancer in the future.
\end{abstract}

\section{Introduction}

Colon cancer is a common malignancy that affects more than 130,000 people each year, causing about 60,000 deaths $[1,2]$. Although the overall five-year survival of patients with colon cancer is generally high with proper treatment, the complex unknown pathogenesis limits the further improvement in colon cancer treatment $[3,4]$. Therefore, there is an urgent need for more insights into the pathogenesis of colon cancer. In recent years, miRNAs' role in cancer research has received increasing attention. miRNA is an important factor in tumorigenesis and metastasis, and its expression characteristics are closely related to the occurrence, progression, and prognosis of various tumors $[5,6]$. Previous studies have identified some important miRNAs impairing the development of cancers by miRNA expression profiles [7-9]. Besides, bioinformatics analysis was widely used for the identification of novel biomarkers and mechanism studies $[10,11]$. In this study, we aimed to search and confirm hub miRNAs that play important parts in the development of colon cancer, thus providing more information for the mechanism research and clinical application of colon cancer.

\section{Materials and Methods}

2.1. Data Collection and Processing. The brief workflow of this study is shown in Figure 1. The microRNA expression profiles of GSE98406, GSE83924, GSE48267, and GSE35834 were downloaded from the Gene Expression Omnibus (GEO) database (https://www.ncbi.nlm.nih.gov/geo/). Quantile normalization was performed to normalize all datasets. Table 


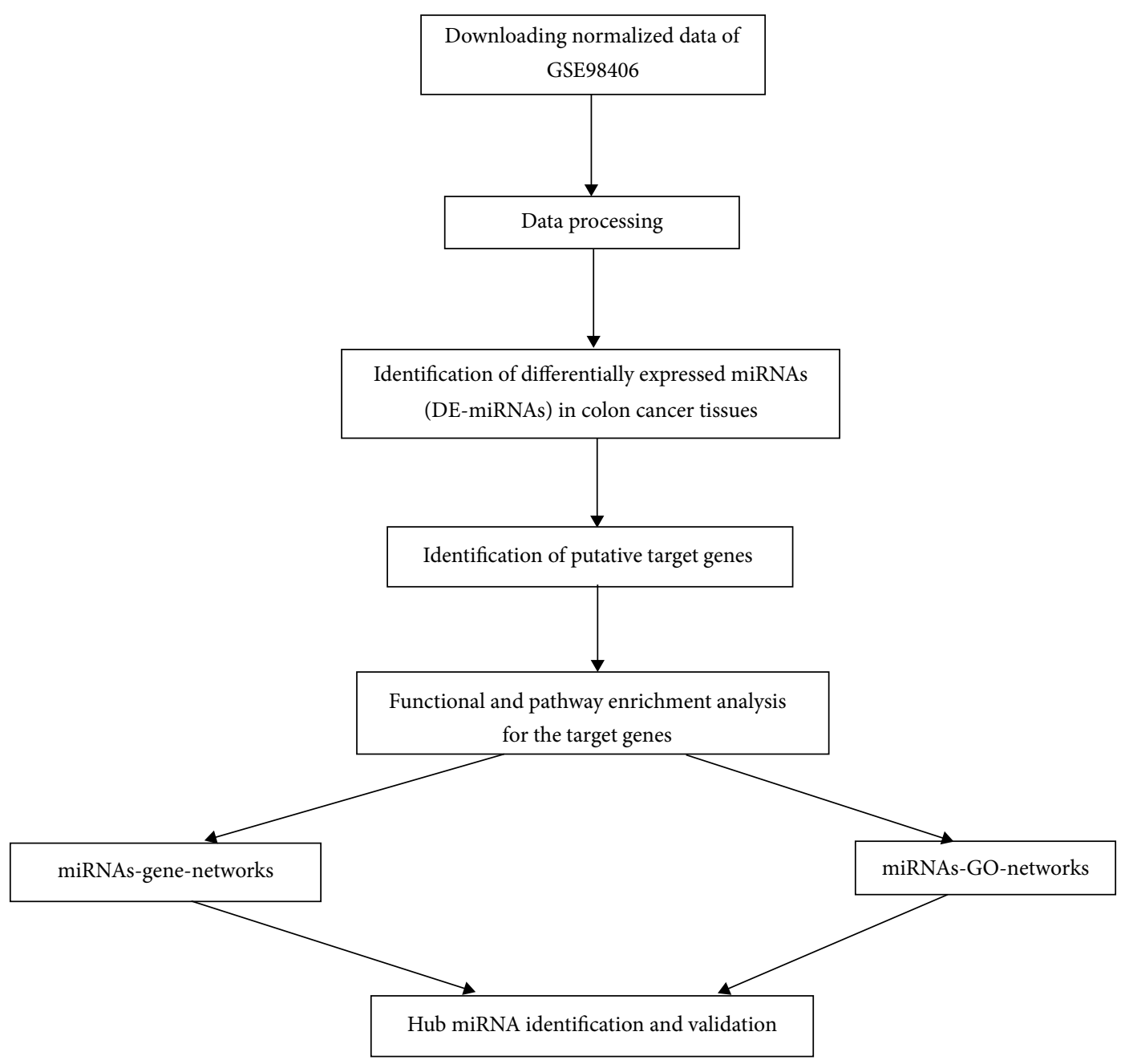

FIGURE 1: Flow chart of data preparation, processing, analysis, and validation.

S1 lists the details of these datasets. GSE98406 was used as a training dataset for screening DE-microRNAs. GSE83924 and GSE48267 were used as independent sample $T$ test sets for verification, respectively. In addition, the clinicopathological correlation analysis for the colon cancer samples in GSE35834 was performed.

2.2. Screening of DE-miRNAs in Colon Cancer Tissues. In this study, the "limma" package in R [12] was used to screen DEmiRNAs between normal colon tissues and tumor tissues. The cutoff criteria were FDR $<0.05$ and $\left|\log _{2} \mathrm{FC}\right|>1.5$.

\subsection{Functional Enrichment Analysis of Putative Target}

Genes. In order to get more knowledge about the candidate miRNAs function, we submitted the selected miRNAs to GCBI to screen their target genes. GCBI is an online tool, which can be used to predict miRNA target genes based on miRanda and TargetScan. Gene Ontology (GO) enrichment analysis and Kyoto Encyclopedia of Genes and Genomes (KEGG) pathway enrichment analysis for target genes were performed. The cut-off criterion is FDR $<0.05$. Also, we drew an interactive network by pathway network (path-net) analysis in GCBI, which covered the significant KEGG pathway to find the hub pathways. The degrees of each pathway in this path-net were calculated, and the top 5 pathways with highest degrees were selected as hub pathways.

2.4. Identification and Validation of HUB miRNAs. After understanding the target genes and the GOs for DE-miRNAs, two important networks for this study (miRNA-gene-network and miRNA-GO-network) were built. Based on the ordering of the number of microRNAs in the two networks, we selected the overlapping hub miRNAs and the key regulatory functions of these miRNA. Two datasets (GSE83924 and GSE48267) were used to verify differential expression levels of these miRNAs between normal colon tissues and tumor tissues by independent sample $T$ test, respectively. $P<0.05$ was considered statistically significant. Meanwhile, the datasets of GSE83924 and GSE48267 were used to perform ROC curve analysis, and the AUC for each hub miRNA was calculated to distinguish the tumor tissues from the normal tissues.

\subsection{The Clinical Significance of HUB miRNAs in Colon} Cancer. Based on 52 cases of colon cancer samples with complete clinical information in GSE35834, the relationship between the expression of hub miRNAs and clinicopathological 


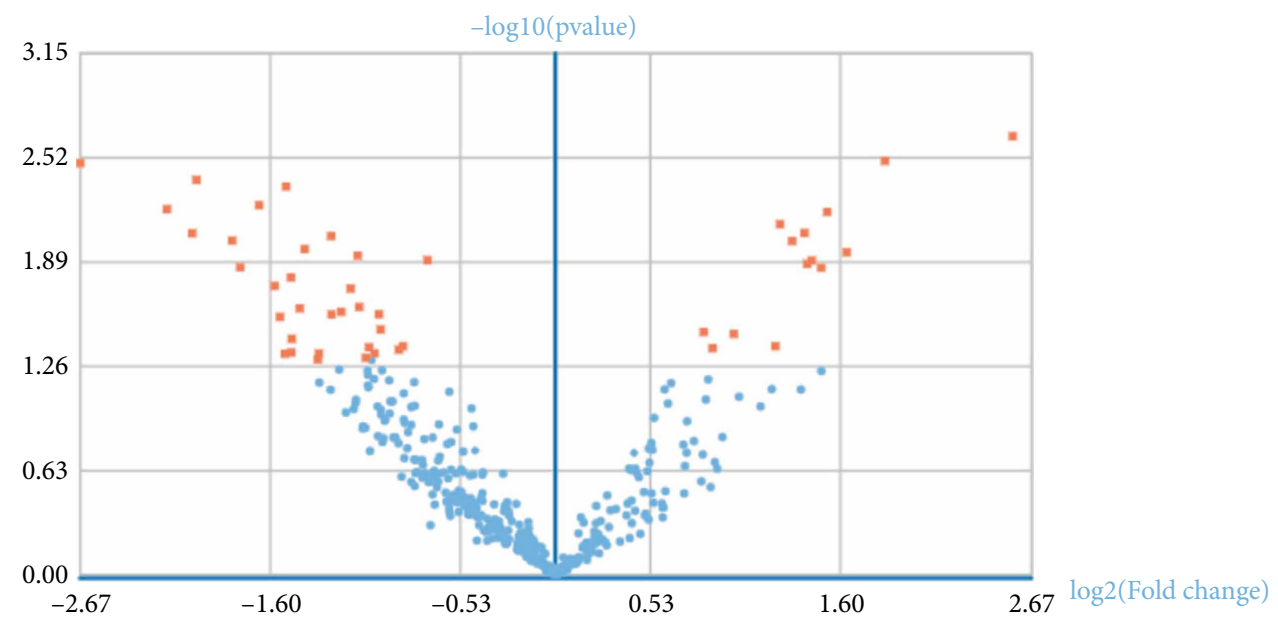

FIgURE 2: The volcano plot for DE-miRNAs.

parameters was evaluated by clinicopathological correlation analysis. According to the amount of hub miRNA expression, 52 cases were classified into high expression group and low expression group according to the hub miRNA expression (high group, $n=26$; low group, $n=26$ ). Chi-square tests were used to assess the relationship between the expression of hub miRNA and gender, age, tumor grade, TNM stage, and metastasis of colon cancer patients. A value of $P<0.05$ was considered as statistically significant.

\section{Results}

3.1. DE-miRNAs and Target Genes in Colon Tumor Tissues. Under the thresholds of FDR $<0.05$ and $\left|\log _{2} \mathrm{FC}\right|>1.5$, a total of 46 DE-miRNAs (14 up-regulated and 32 downregulated in colon cancer samples) were selected from 7 control samples and 14 colon samples in GSE98406. The volcano plot for DE-miRNAs was taken (Figure 2) and the characteristics of the dysregulated miRNAs are listed in Table 1. Based on miRanda and TargetScan, 1738 putative miRNA target genes were identified using GCBI (Table S2).

3.2. Functional Enrichment Analysis of Target Genes. To study the roles of DE-miRNAs in mediating colon cancer progression, we performed GO analysis and KEGG pathway enrichment analysis for target genes. The data in Table 2 indicate that top 10 GOs were "transcription, DNA-dependent", "regulation of transcription, DNA-dependent", "signal transduction", "positive regulation of transcription from RNA polymerase II promoter", "apoptotic process", "positive regulation of transcription, DNA-dependent", "negative regulation of transcription from RNA polymerase II promoter", "nervous system development", "axon guidance" and "protein phosphorylation". According to the KEGG database, the main pathways involving the target genes were demonstrated. As shown in Table 3, the top 10 pathways were "MAPK signaling pathway", "pathways in cancer", "PI3K-Akt signaling pathway, proteoglycans in cancer", "HTLV-I infection", "endocytosis", "transcriptional mis-regulation in cancer", "neurotrophin signaling pathway",

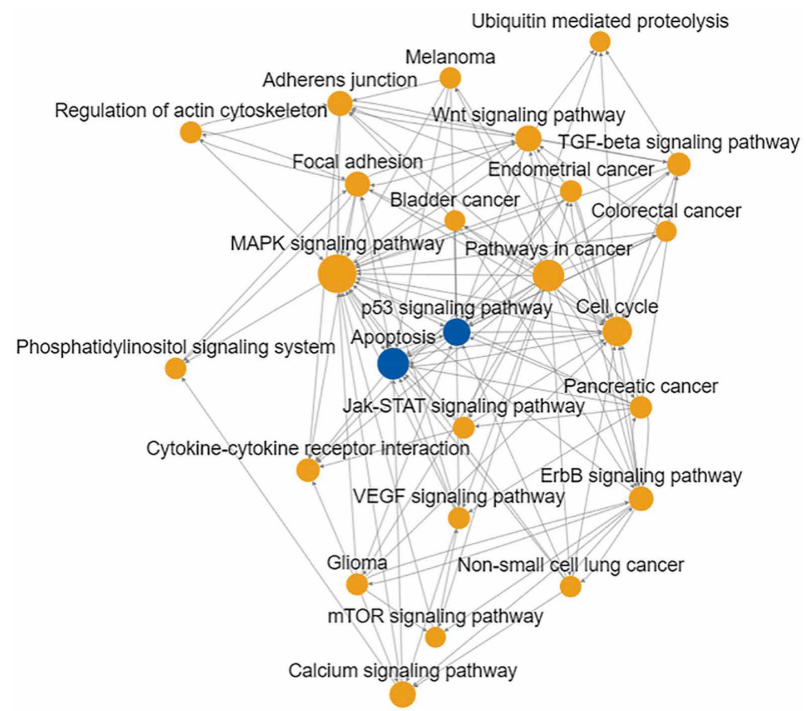

Figure 3: Pathway network (Path-net). Significantly changed pathways were connected in a Path-net to show the interaction network among these pathways. Each pathway in the network was measured by counting the upstream and downstream pathways. The blue circle represents pathways involving upregulated miRNAs, while the yellow circle represents pathways involving both upregulated and downregulated miRNAs. The size of the circle represents the degree value and the lines show the interaction between pathways. A higher degree of pathway indicates that it plays a more important role in the signaling network.

"axon guidance and GnRH signaling pathway". What is more, a pathway network was shown, which covers 25 significantly changed pathways (Figure 3). Moreover, the MAPK signaling pathway (degrees:41), apoptosis (degrees:27), pathways in cancer (degree:27), cell cycle (degrees:23) and p53 signaling pathway (degrees:23) showed highest connectivity degrees in path-net, which demonstrated these 5 pathways would play a central role in tumor development.

3.3. Hub miRNAs Identification and Validation. To identify hub miRNAs and their main functions in the development 
TABLE 1: The characteristics of the dysregulated miRNAs.

\begin{tabular}{|c|c|c|c|c|}
\hline Rank & miRNAs & FC & FDR & Feature \\
\hline 1 & miR-486-5p & 5.903792 & 0.002243 & up \\
\hline 2 & miR-451a & 3.594096 & 0.003163 & up \\
\hline 3 & miR-378i & 2.871432 & 0.006424 & up \\
\hline 4 & miR-126-3p & 2.390553 & 0.007611 & up \\
\hline 5 & miR-378a-3p & 2.628745 & 0.008606 & up \\
\hline 6 & miR-378c & 2.505525 & 0.009621 & up \\
\hline 7 & miR-30a-5p & 3.096433 & 0.011253 & up \\
\hline 8 & miR-378f & 2.70463 & 0.012609 & up \\
\hline 9 & $\operatorname{miR}-422 a$ & 2.655992 & 0.013226 & up \\
\hline 10 & miR-139-5p & 2.804786 & 0.013957 & up \\
\hline 11 & miR-1280 & 1.776806 & 0.034109 & up \\
\hline 12 & miR-4286 & 1.997162 & 0.034955 & up \\
\hline 13 & miR-193b-3p & 2.347341 & 0.041491 & up \\
\hline 14 & miR-125a-5p & 1.83864 & 0.042671 & up \\
\hline 15 & miR-182-5p & -6.34791 & 0.003257 & down \\
\hline 16 & miR-3687 & -4.03913 & 0.004112 & down \\
\hline 17 & miR-503-5p & -2.85125 & 0.004506 & down \\
\hline 18 & miR-18b-5p & -3.16775 & 0.005848 & down \\
\hline 19 & miR-4417 & -4.53068 & 0.006188 & down \\
\hline 20 & miR-1246 & -4.10737 & 0.00864 & down \\
\hline 21 & miR-224-5p & -2.39326 & 0.008975 & down \\
\hline 22 & miR-200c-3p & -3.51686 & 0.009565 & down \\
\hline 23 & miR-552-3p & -2.65361 & 0.010763 & down \\
\hline 24 & miR-877-5p & -2.15946 & 0.011788 & down \\
\hline 25 & miR-501-5p & -1.64608 & 0.012554 & down \\
\hline 26 & miR-203a & -3.40787 & 0.013864 & down \\
\hline 27 & miR-146a-5p & -2.79816 & 0.015957 & down \\
\hline 28 & miR-18a-5p & -2.98152 & 0.017964 & down \\
\hline 29 & miR-210-3p & -2.21905 & 0.018626 & down \\
\hline 30 & miR-424-3p & -2.14658 & 0.024091 & down \\
\hline 31 & miR-1290 & -2.70448 & 0.024521 & down \\
\hline 32 & miR-25-5p & -2.3023 & 0.025739 & down \\
\hline 33 & miR-4449 & -1.98777 & 0.026635 & down \\
\hline 34 & miR-3651 & -2.38984 & 0.026706 & down \\
\hline 35 & miR-141-3p & -2.92073 & 0.027618 & down \\
\hline 36 & miR-17-5p & -1.97557 & 0.032908 & down \\
\hline 37 & miR-200a-3p & -2.79035 & 0.037529 & down \\
\hline 38 & miR-188-5p & -1.81097 & 0.04154 & down \\
\hline 39 & miR-106b-3p & -2.06644 & 0.042212 & down \\
\hline 40 & miR-130b-3p & -1.84017 & 0.043567 & down \\
\hline 41 & miR-21-5p & -2.79495 & 0.045308 & down \\
\hline 42 & miR-19a-3p & -2.02304 & 0.045882 & down \\
\hline 43 & miR-3648 & -2.51032 & 0.045897 & down \\
\hline 44 & miR-708-5p & -2.86582 & 0.046121 & down \\
\hline 45 & miR-155-5p & -2.09222 & 0.048755 & down \\
\hline 46 & miR-3175 & -2.52047 & 0.04994 & down \\
\hline
\end{tabular}

of colon cancer, we selected target genes and DE-miRNAs to construct miRNAs-gene-networks (Figure 4) and miRNAsGO-networks (Figure 5) according to the significant regulation of GOs and pathways. According to the rank of degrees of miRNAs in two networks, the top rated three miRNAs (miR-17-5p, miR-182-5p, and miR-200a-3p) were determined

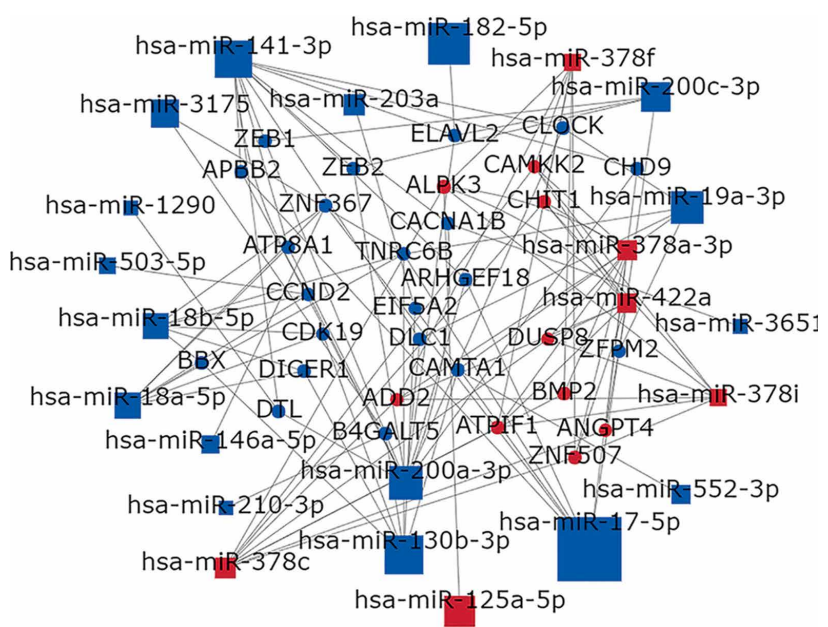

FIgURE 4: miRNAs-gene-network. According to the interactions between miRNAs and the intersected target genes, miRNAs-genenetwork was constructed. The blue circles represent genes, while blue square nodes represent downregulated miRNAs. The size of the circle or square node represents the degree value. A higher degree of gene/ miRNAs indicates that it plays a more important role in the signaling network.

to be hub miRNAs (Table 4). The bioinformatic analysis showed the hub miRNAs were lowly expressed in colon cancer tissues compared with normal colon tissues. And dysregulated miRNAs play important roles in signal transduction, apoptotic process, and pathways in cancer. GSE83924 and GSE48267 were used to make validation. Further proved by the datasets of GSE83924 and GSE48267, miR-17-5p, miR-182-5p and miR-200a-3p were lowly expressed in tumor tissues (Figure 6). Besides, ROC curve for GSE83924 indicated that miR-17-5p $(\mathrm{AUC}=0.918)$, miR-182-5p $(\mathrm{AUC}=0.853)$ and $\mathrm{miR}-200 \mathrm{a}-3 \mathrm{p}$ $(\mathrm{AUC}=0.783)$ exhibited excellent diagnostic efficiency for tumor and normal tissues. And ROC curve for GSE48267 also demonstrated that miR-17-5p (AUC=0.829), miR-182-5p $(\mathrm{AUC}=0.849)$ and miR-200a-3p $(\mathrm{AUC}=0.709)$ exhibited diagnostic efficiency for tumor and normal tissues (Figure 7).

3.4. Association of Hub miRNAs Expression with Clinical Significance. Chi-square analysis for GSE35834 showed that miR-17-5p expression was associated with tumor grade significantly $(P=0.02)$, and miR-182-5p expression group was associated with advanced TNM stage (III/IV) $(P=0.019)$. No other significant difference was observed in other clinicopathological features (age, gender, and metastasis) (Table S3).

\section{Discussion}

In this study, bioinformatics analysis of GSE98406 revealed 46 DE-miRNAs (down-regulated 32 and up-regulated 14). According to the miRNAs-gene-networks and miRNAs-GOnetworks, miR-17-5p, miR-182-5p, and miR-200a-3p were considered to be hub miRNAs. They play an important role in tumor development as tumor suppressor genes and oncogenes. Although miR-182-5p and miR-200a-3p have 
TABLE 2: The top 10 dysregulated GOs.

\begin{tabular}{llccc}
\hline Rank & GO ID & GO name & Count & FDR \\
\hline 1 & GO:0006351 & Transcription, DNA-dependent & 278 & $1.54 \mathrm{E}-60$ \\
2 & GO:0006355 & Regulation of transcription, DNA-dependent & 169 & $3.34 \mathrm{E}-27$ \\
3 & GO:0007165 & Signal transduction & 165 & $1.81 \mathrm{E}-37$ \\
4 & GO:0045944 & Positive regulation of transcription from RNA polymerase II promoter & 154 & $5.61 \mathrm{E}-52$ \\
5 & GO:0006915 & Apoptotic process & 111 & $4.75 \mathrm{E}-27$ \\
6 & GO:0045893 & Positive regulation of transcription, DNA-dependent & 103 \\
7 & GO:0000122 & Negative regulation of transcription from RNA polymerase II promoter & $1.14 \mathrm{E}-32$ \\
8 & GO:0007399 & Nervous system development & $2.41 \mathrm{E}-31$ \\
9 & GO:0007411 & Axon guidance & 75 \\
10 & GO:0006468 & Protein phosphorylation & 75 & $4.70 \mathrm{E}-30$ \\
\hline
\end{tabular}

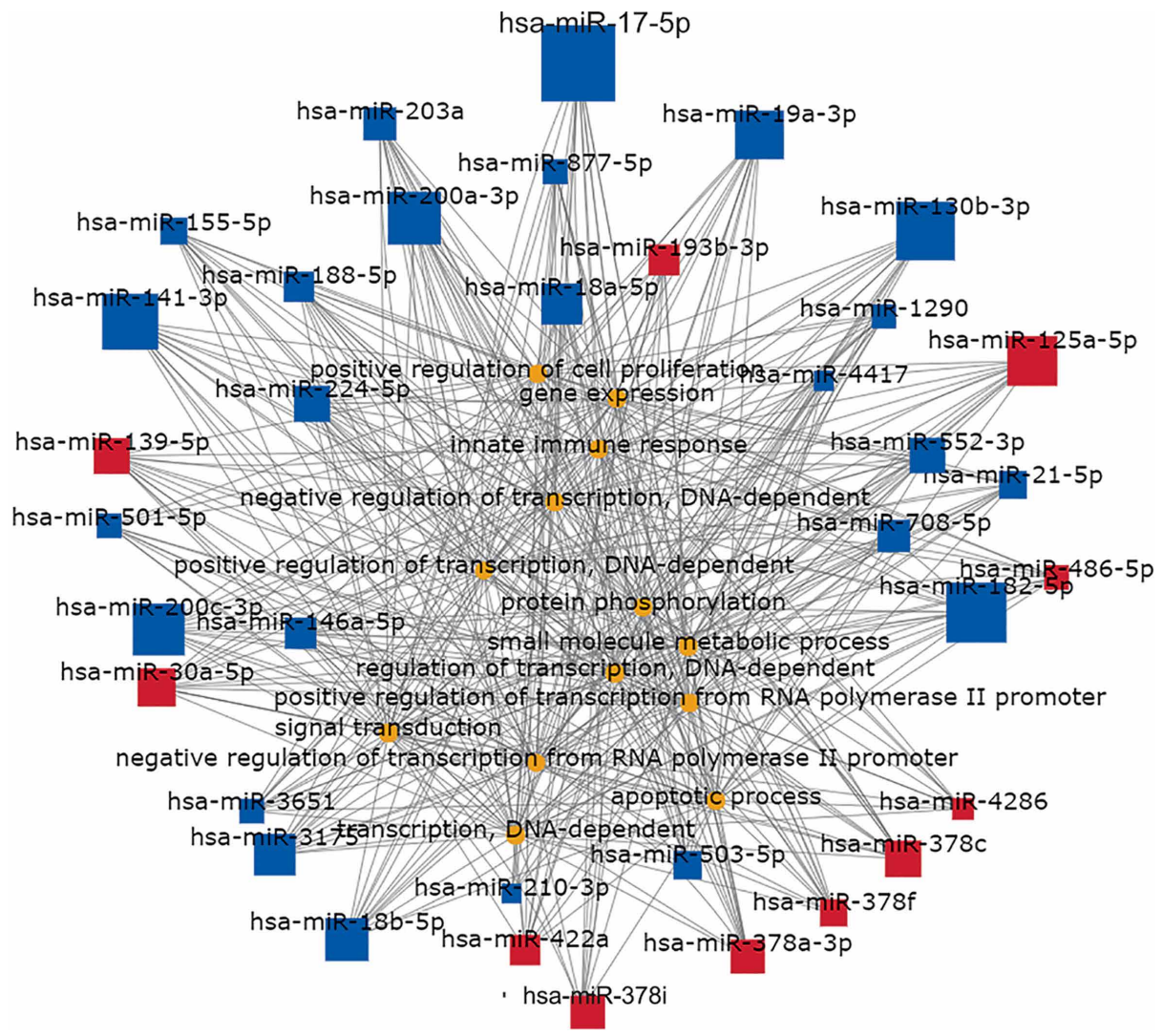

FIGURE 5: miRNAs-GO-network. The miRNAs-GO-network was generated according to the relationship of significant biological functions and miRNAs. The yellow and blue circles represent GOs, red square nodes represent upregulated miRNAs, and blue square nodes represent downregulated miRNAs. The size of the circle or square node represents the degree value. A higher degree of GO/miRNAs indicates that it plays a more important role in the signaling network.

been found to be associated with colorectal cancer [13-15], there is still a lack of relevant studies exploring its regulatory mechanisms in colon cancer. As for miR-17-5, it is the first time to discover it was negatively related to tumor progression.

Then, using the target prediction method in the GCBI online tool, 1738 genes were selected as target genes for these DE-miRNAs. The target genes predicted by GO analysis were enriched in "transcription, DNA-dependent", "transcriptional regulation, DNA-dependent", "signal transduction" and "positive regulation of transcription from RNA polymerase II promoter". Interestingly, we noticed the opposite GOs (negative regulation of transcription from RNA polymerase II promoter and Positive regulation of transcription from RNA polymerase II promoter). Taken together, the hub miRNAs (hsa-miR-17-5p, hsa-miR-182-5p and hsa-miR-200a-3p) that we identified were reliable, which may be candidate biomarkers for colon cancer.

As for the 3 hub miRNAs, we conducted a literature review of these miRNAs. miR-17-5p is an important regulator, which has a strong effect on the G1/S phase of cell cycle transition [16]. MiR-17 has been found to target certain genes in some cancers, such as bladder cancer and oral squamous cell 


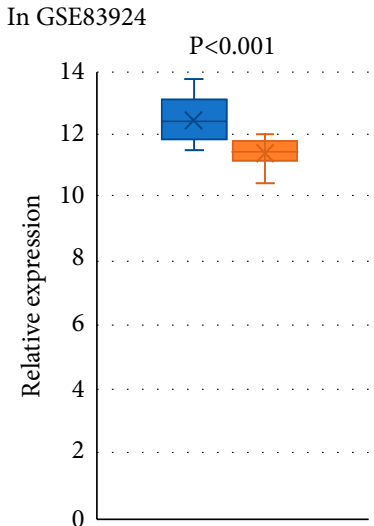

(a)

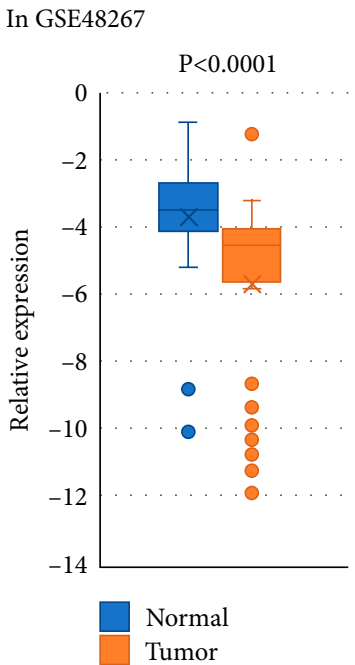

(d)

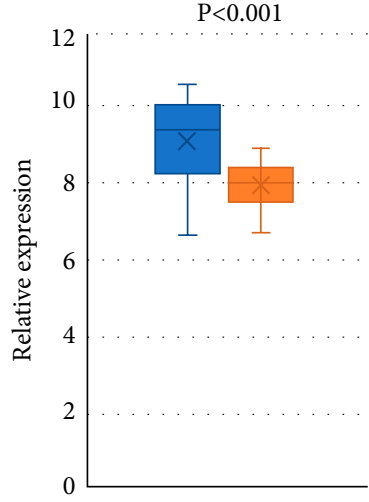

(b)

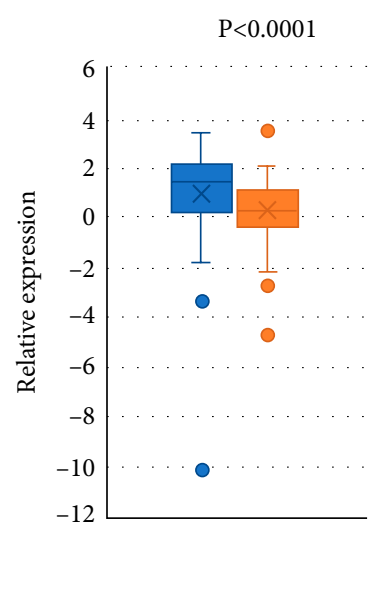

(e)

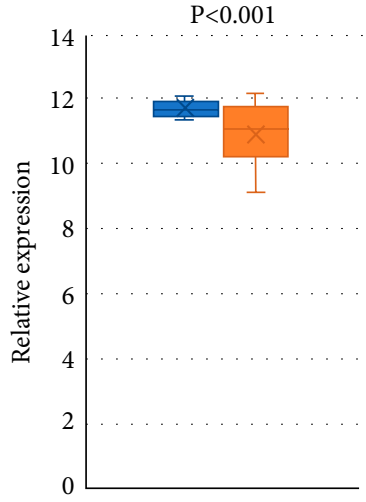

(c)

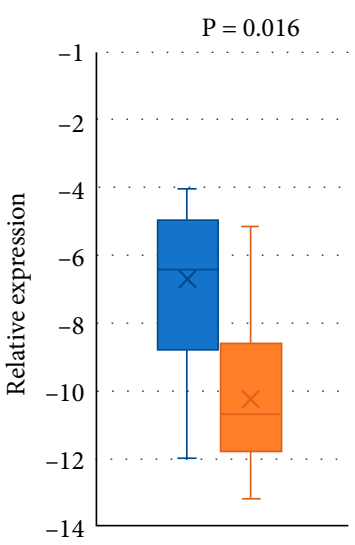

(f)

Figure 6: In GSE83924, miRNAs expression levels of miR-17-5p (a), miR-182-5p (b), and miR-200a-3p (c) between normal colon and tumor samples. In GSE48267, miRNAs expression levels of miR-17-5p (d), miR-182-5p (e), and miR-200a-3p (f) between normal colon and tumor samples. Independent sample $T$ test was used to evaluate the statistical significance of differences.

TABLE 3: The top ten dysregulated pathways of the target genes of DE-miRNAs.

\begin{tabular}{|c|c|c|c|c|}
\hline Rank & Pathway ID & Pathway name & Count & FDR \\
\hline 1 & 04010 & $\begin{array}{l}\text { MAPK signaling } \\
\text { pathway }\end{array}$ & 69 & $2.00 \mathrm{E}-28$ \\
\hline 2 & 05200 & $\begin{array}{c}\text { Pathways in } \\
\text { cancer }\end{array}$ & 64 & $8.44 \mathrm{E}-19$ \\
\hline 3 & 04151 & $\begin{array}{c}\text { PI3K-Akt } \\
\text { signaling pathway }\end{array}$ & 61 & $1.17 \mathrm{E}-15$ \\
\hline 4 & 05205 & $\begin{array}{l}\text { Proteoglycans in } \\
\text { cancer }\end{array}$ & 47 & $5.80 \mathrm{E}-15$ \\
\hline 5 & 05166 & HTLV-I infection & 47 & $2.77 \mathrm{E}-12$ \\
\hline 6 & 04144 & Endocytosis & 45 & $2.58 \mathrm{E}-15$ \\
\hline 7 & 05202 & $\begin{array}{l}\text { Transcriptional } \\
\text { misregulation in } \\
\text { cancer }\end{array}$ & 38 & $1.97 \mathrm{E}-12$ \\
\hline 8 & 04722 & $\begin{array}{c}\text { Neurotrophin } \\
\text { signaling pathway }\end{array}$ & 33 & $3.08 \mathrm{E}-14$ \\
\hline 9 & 04360 & Axon guidance & 31 & $1.38 \mathrm{E}-11$ \\
\hline 10 & 04912 & $\begin{array}{c}\text { GnRH signaling } \\
\text { pathway }\end{array}$ & 24 & $6.40 \mathrm{E}-10$ \\
\hline
\end{tabular}

TABLE 4: Hub miRNAs in miRNAs-gene-networks and miRNAs-GO-networks.

\begin{tabular}{lcccc}
\hline Rank & miRNAs & Feature & $\begin{array}{c}\text { miRNA-gene- } \\
\text { networks } \\
\text { degree }\end{array}$ & $\begin{array}{c}\text { miRNA-GO- } \\
\text { networks } \\
\text { degree }\end{array}$ \\
\hline 1 & miR-17-5p & down & 277 & 449 \\
2 & miR-182-5p & down & 177 & 405 \\
3 & miR-200a-3p & down & 126 & 342 \\
\hline
\end{tabular}

carcinoma $[17,18]$. miR-182-5p is a member of the miR183/96/182 cluster. Previous studies have identified its important role in breast cancer, glioma, prostate cancer, prostate cancer and renal cell carcinoma [19-23]. Generally, miR-182-5p regulates the apoptosis of tumor cells by targeting certain special genes, such as FOXO1, MTSS1, HMGA2, CASP9, and FOXO3 $[24,25]$, and these target genes were also predicted by our experiment. miR-200a-3p has been found to play important roles in the epithelial to the mesenchymal transition process in the development of cancer $[26,27]$. 

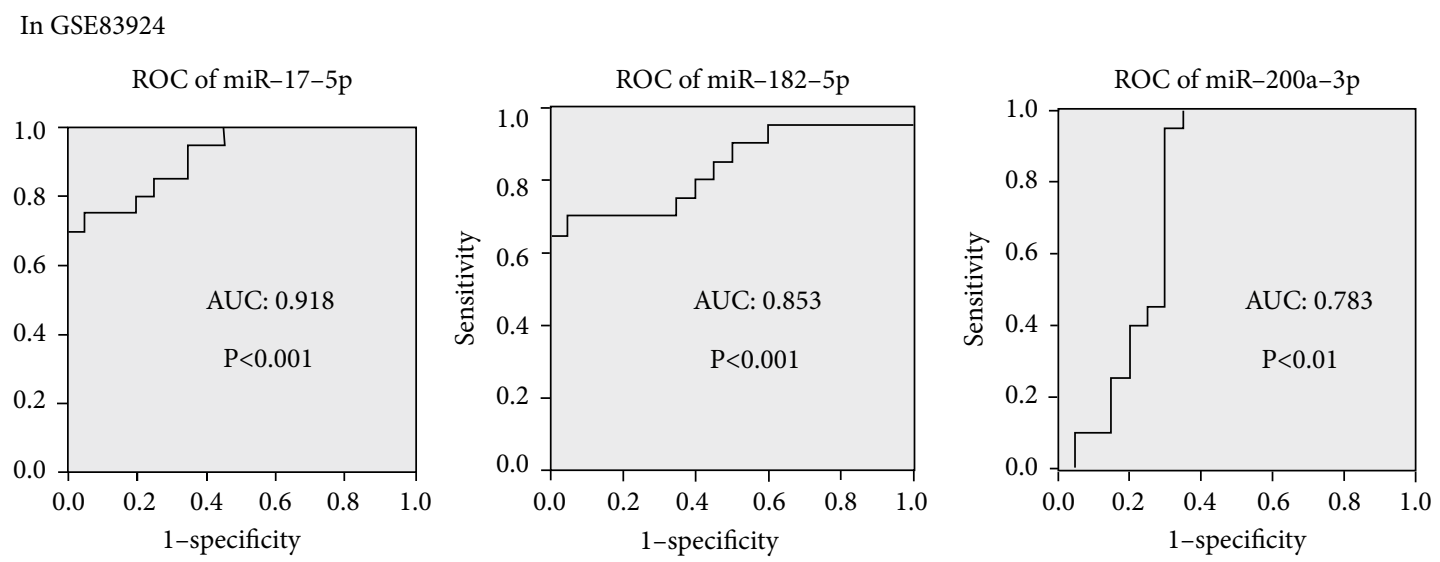

\section{In GSE48267}
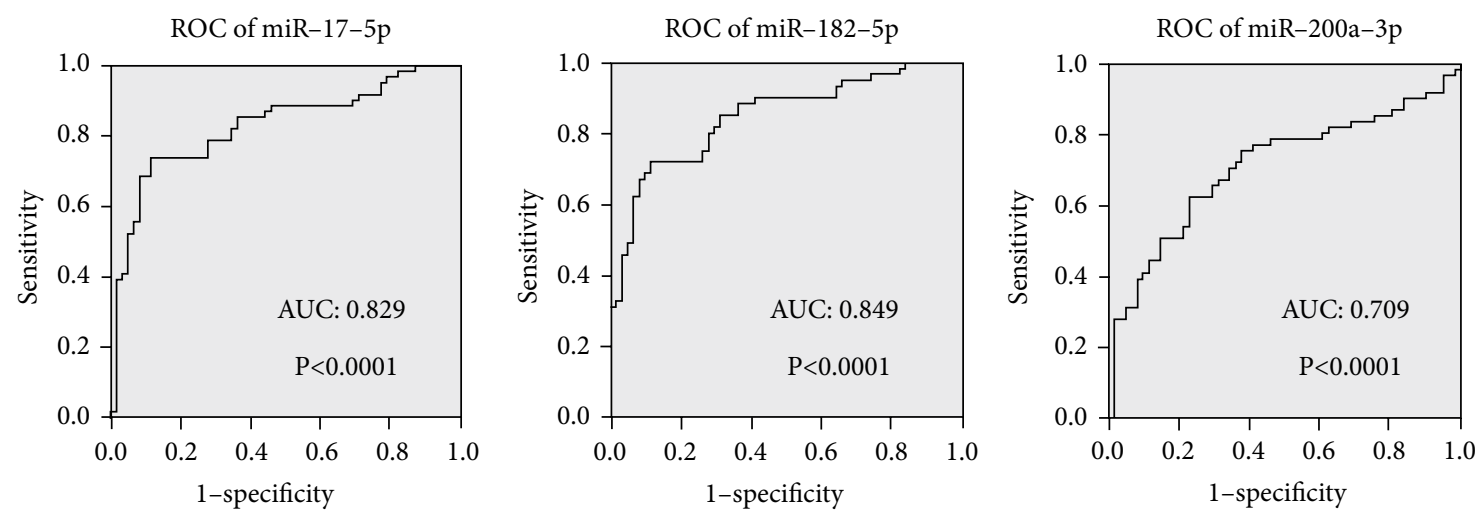

FIGURE 7: ROC curve of miR-17-5p, miR-182-5p and miR-200a-3p in the datasets of GSE83924 and GSE48267.

miR-200a-3p plays a role like a tumor suppressor and its target genes are enriched in signal transmission and cell apoptosis control. miR-200a-3p is rarely used as a research focus and related regulatory mechanisms remain to be clarified.

In summary, we identified three hub miRNAs (hsa-miR17-5p, hsa-miR-182-5p, and hsa-miR-200a-3p), which were closely related to the development of colon cancer. The hub miRNAs we identified might provide references for the functional study of downstream proteins in other study and some clinical targeted treatments in the future. However, due to the small sample size of this study, these results still have certain limitations. Further, in vivo and in vitro studies are needed to understand the exact molecular mechanisms that influence the development of colon cancer.

\section{Data Availability}

The data supporting the results reported in this article can be available by contacting the corresponding author.

\section{Conflicts of Interest}

The authors declare that they have no conflicts of interest.

\section{Authors' Contributions}

Dong-Hu Yu and Wei Li are co-first authors. X.R., and S.L. conceived and designed the study, D.Y., W.L., and X.L. performed the analysis procedures, D.Y., W.L., J.H., C.Z., and X.L. analyzed the results, X.L., X.R., and S.L. contributed analysis tools, D.Y., and W.L. contributed to the writing of the manuscript. All authors reviewed the manuscript.

\section{Funding}

This work was supported by the 351 Talent Project of Wuhan University (Luojia Young Scholars: SL) and Young \& Middleaged Medical Key Talents Training Project of Wuhan.

\section{Supplementary Materials}

Supplementary 1. Table S1: the details of GSE98406, GSE83924, GSE48267 and GSE35834.

Supplementary 2. Table S2: putative target genes of DEmiRNAs.

Supplementary 3. Table S3: clinicopathological correlation analysis for GSE35834. 


\section{References}

[1] R. Siegel, C. DeSantis, and A. Jemal, "Colorectal cancer statistics, 2014," CA: A Cancer Journal for Clinicians, vol. 64, no. 2, pp. 104-117, 2014.

[2] R. L. Siegel, K. D. Miller, S. A. Fedewa et al., "Colorectal cancer statistics, 2017," CA: A Cancer Journal for Clinicians, vol. 67, no. 3, pp. 177-193, 2017.

[3] B. Liang, M. Shahbaz, Y. Wang et al., "Integrin beta 6-targeted immunoliposomes mediate tumor-specific drug delivery and enhance therapeutic efficacy in colon carcinoma," Clinical Cancer Research, vol. 21, no. 5, pp. 1183-1195, 2015.

[4] S. Mannucci, L. Ghin, G. Conti et al., "Magnetic nanoparticles from magnetospirillum gryphiswaldense increase the efficacy of thermotherapy in a model of colon carcinoma," PLoS One, vol. 9, no. 10, p. e108959, 2014.

[5] Y. He, J. Lin, D. Kong et al., "Current state of circulating microRNAs as cancer biomarkers," Clinical Chemistry, vol. 61, no. 9, pp. 1138-1155, 2015.

[6] J.P.S. Chawla, N. Iyer, K. S. Soodan, A. Sharma, S. K. Khurana, and P. Priyadarshni, "Role of miRNA in cancer diagnosis, prognosis, therapy and regulation of its expression by Epstein-Barr virus and human papillomaviruses: with special reference to oral cancer," Oral Oncology, vol. 51, no. 8, pp. 731-737, 2015.

[7] J. W. F. Catto, S. Miah, H. C. Owen et al., "Distinct microRNA alterations characterize high- and low-grade bladder cancer," Cancer Research, vol. 69, no. 21, pp. 8472-8481, 2009.

[8] G. Pignot, G. Cizeron-Clairac, S. Vacher et al., "microRNA expression profile in a large series of bladder tumors: identification of a 3-miRNA signature associated with aggressiveness of muscleinvasive bladder cancer," International Journal of Cancer, vol. 132, no. 11, pp. 2479-2491, 2013.

[9] H. Yoshino, N. Seki, T. Itesako, T. Chiyomaru, M. Nakagawa, and H. Enokida, "Aberrant expression of microRNAs in bladder cancer," Nature Reviews Urology, vol. 10, no. 7, pp. 396-404, 2013.

[10] X.-Y. Meng, M.-J. Shi, Z.-H. Zeng et al., “The role of COL5A2 in patients with muscle-invasive bladder cancer: bioinformatics analysis of public datasets involving 787 subjects and 29 cell lines," Frontiers in Oncology, vol. 8, 2019.

[11] X. Wang, L. Chen, H. Jin et al., "Screening miRNAs for early diagnosis of colorectal cancer by small RNA deep sequencing and evaluation in a Chinese patient population," Oncotargets and Therapy, vol. 9, pp. 1159-1166, 2016.

[12] M. E. Ritchie, B. Phipson, D. Wu et al., "Limma powers differential expression analyses for RNA-sequencing and microarray studies," Nucleic Acids Research, vol. 43, no. 7, p. e47, 2015.

[13] Y. Li, Y. Lu, and Y. Chen, "Long non-coding RNA SNHG16 affects cell proliferation and predicts a poor prognosis in patients with colorectal cancer via sponging miR-200a-3p," Bioscience Reports, vol. 39, no. 5, Article ID BSR20182498, 2019.

[14] Y. Jin, Z. L. Zhang, Y. Huang, K. N. Zhang, and B. Xiong, "miR$182-5 \mathrm{p}$ inhibited proliferation and metastasis of colorectal cancer by targeting MTDH," European Review for Medical and Pharmacological Sciences, vol. 23, no. 4, pp. 1494-1501, 2019.

[15] M.-Q. Cao, A.-B. You, X.-D. Zhu et al., "miR-182-5p promotes hepatocellular carcinoma progression by repressing FOXO3a," Journal of Hematology \& Oncology, vol. 11, no. 1, 2018.
[16] N. Cloonan, M. K. Brown, A. L. Steptoe et al., "The miR-17$5 p$ microRNA is a key regulator of the G1/S phase cell cycle transition," Genome Biology, vol. 9, no. 8, p. R127, 2008.

[17] F. Wang, D. Wu, Z. Xu et al., "miR-182-5p affects human bladder cancer cell proliferation, migration and invasion through regulating Cofilin 1," Cancer Cell International, vol. 19, no. 1, 2019.

[18] Y. Guo, X. Qiao, L. Zhu, and R. Song, "MicroRNA-182-5p modulates oral squamous cell carcinoma migration and invasion via targeting MTSS1 gene," Pathology \& Oncology Research, 2019.

[19] M. Sharifi and A. Moridnia, "Apoptosis-inducing and antiproliferative effect by inhibition of miR-182-5p through the regulation of CASP9 expression in human breast cancer," Cancer Gene Therapy, vol. 24, no. 2, pp. 75-82, 2017.

[20] J. Xue, A. Zhou, Y. Wu et al., "miR-182-5p induced by STAT3 activation promotes glioma tumorigenesis," Cancer Research, vol. 76, no. 14, pp. 4293-4304, 2016.

[21] J. Yao, C. Xu, Z. Fang et al., "Androgen receptor regulated microRNA miR-182-5p promotes prostate cancer progression by targeting the ARRDC3/ITGB4 pathway," Biochemical and Biophysical Research Communications, vol. 474, no. 1, pp. 213-219, 2016.

[22] K. Tsuchiyama, H. Ito, M. Taga et al., "Expression of microRNAs associated with Gleason grading system in prostate cancer: miR-182-5p is a useful marker for high grade prostate cancer," The Prostate, vol. 73, no. 8, pp. 827-834, 2013.

[23] X. Xu, J. Wu, S. Li et al., "Downregulation of microRNA-182-5p contributes to renal cell carcinoma proliferation via activating the AKT/FOXO3a signaling pathway," Molecular Cancer, vol. 13, no. 1, p. 109, 2014.

[24] H. Hirata, K. Ueno, V. Shahryari et al., "MicroRNA-182-5p promotes cell invasion and proliferation by down regulating FOXF2, RECK and MTSS1 genes in human prostate cancer," PLoS One, vol. 8, no. 1, Article ID e55502, 2013.

[25] L. Yao, Q. Zhou, L. Wang, and G. Hou, "MicroRNA-182-5p protects $\mathrm{H} 9 \mathrm{c} 2$ cardiomyocytes from hypoxia-induced apoptosis by down-regulation of PTEN," International Journal of Clinical and Experimental Pathology, vol. 10, no. 5, pp. 5220-5226, 2017.

[26] Y. Sato, H. Kobayashi, Y. Suto et al., "Chromosomal instability in chromosome band 12p13: multiple breaks leading to complex rearrangements including cytogenetically undetectable sub-clones," Leukemia, vol. 15, no. 8, pp. 1193-1202, 2001.

[27] O. Saydam, Y. Shen, T. Wuerdinger et al., "Downregulated MicroRNA-200a in meningiomas promotes tumor growth by reducing E-Cadherin and activating the Wnt/beta-Catenin signaling pathway," Molecular and Cellular Biology, vol. 29, no. 21, pp. 5923-5940, 2009. 